ARTIGO ORIGINAL

\title{
Modelos de crescimento e produção para a estimativa volumétrica em povoamentos comerciais de eucalipto
}

\author{
Growth and production models for volumetric estimates in commercial \\ eucalypt stands \\ Tamires Mousslech Andrade Penido ${ }^{1}$ (B), Bruno Oliveira Lafetá ${ }^{2}$ (D), Gilciano Saraiva Nogueira ${ }^{1}$ (D), \\ Petrônio Henrique Alves ${ }^{1}$ (D), Eric Bastos Gorgens ${ }^{1}$ (D), Marcio Leles Romarco de Oliveira ${ }^{1}$ (D) \\ ${ }^{1}$ Universidade Federal dos Vales do Jequitinhonha e Mucuri - UFVJM, Diamantina, MG, Brasil \\ ${ }^{2}$ Instituto Federal de Educação Ciência e Tecnologia Minas Gerais - IFMG, São João Evangelista, MG, Brasil
}

Como citar: Penido, T. M. A., Lafetá, B. O., Nogueira, G. S., Alves, P. H., Gorgens, E. B., \& Oliveira, M. L. R. (2020) Modelos de crescimento e produção para a estimativa volumétrica em povoamentos comerciais de eucalipto. Scientia Forestalis, 48(128), e3340. https://doi.org/10.18671/scifor.v48n128.06

\begin{abstract}
Resumo
O objetivo deste estudo foi avaliar três categorias de modelos de crescimento e produção para a estimativa volumétrica em povoamentos comerciais de eucalipto. Os dados foram provenientes de inventário florestal contínuo, obtidos em 34 parcelas permanentes de $400 \mathrm{~m} 2$, em quatro unidades de manejo, que totalizaram 293,43 ha. As categorias de modelo testadas foram: em nível de povoamento (MP), pelo sistema de equações simultâneas de Clutter; de distribuição diamétrica (MDD), pelo ajuste de função densidade de probabilidade de Weibul-2P e de árvores individuais (MAI), pelo modelo de Pienaar e Schiver. As três categorias de modelo proporcionaram estimativas confiáveis de volume com casca, aos 36, 48, 60 e 72 meses. Embora as projeções com MP e MDD tenham gerado estimativas similares de volume para idades além daquelas em que se realizou o inventário florestal, o MAl foi a categoria mais precisa e consistente na quantificação do volume por hectare.
\end{abstract}

Palavras-chave: Clutter; Modelo de árvores individuais; Modelo de distribuição de diâmetros; Pienaar e Schiver.

\begin{abstract}
The aim of this study was to evaluate three categories of growth and production models for volumetric estimation in commercial eucalypt stands. A continuous forest inventory was performed in 34 permanent plots of $400 \mathrm{~m}^{2}$, in four management units, which totaled $293.43 \mathrm{ha}$. Model categories tested were: at the stand level (MP), by the simultaneous equations system of Clutter; diameter distribution model (MDD), by the adjustment of the Weibull-2P's probability density function and individual trees (MAl), by the Pienaar and Schiver model. The three model categories provided reliable estimates of volume with bark at $36,48,60$ and 72 months. Although projections with MP and MDD generated similar volume estimates for ages beyond those in which the forest inventory was performed, MAI was the most accurate and consistent category in volume quantification per hectare.
\end{abstract}

Keywords: Clutter; Model of individual trees; Model of diameters distribution; Pienaar and Schiver.

Fonte de financiamento: CNPq, UFVJ.M

Conflito de interesse: Nada a declarar.

Autor correspondente: penidotma@gmail.com

Recebido: 17 abril 2019.

Aceito: 18 outubro 2019.

Editor: Paulo Henrique Müller Silva.

(c) Este é um artigo publicado em acesso aberto (Open Access) sob a licença Creative Commons Attribution, que permite uso, distribuição e sł reprodução em qualquer meio, sem restrições desde que o trabalho original seja corretamente citado. 


\section{INTRODUÇÃO}

Os projetos florestais são caracterizados pelo longo período entre os investimentos iniciais e a receita futura, o que provoca grande expectativa sobre o volume a ser colhido (Demolinari et al., 2007). As estimativas volumétricas devem ser precisas para melhor eficiência logística das atividades do setor e atendimento da demanda industrial madeireira. Ferramentas para o prognóstico do estoque de madeira e o rendimento são necessárias para um planejamento estratégico adequado (Vanclay, 1994; Trincado et al., 2003; Diamantopoulou et al., 2015).

A modelagem é um procedimento estatístico empregado por gestores para esboçar o desenvolvimento vegetal com confiabilidade, auxiliando na definição de tratamentos silviculturais e do momento ideal para o corte, compra e venda da madeira ou aquisição de novas áreas (Vanclay, 1994; Nascimento et al., 2015; Pereira et al., 2016). As três principais categorias de modelos de crescimento e produção são destinadas para povoamento, distribuição diamétrica ou árvores individuais (Davis et al., 2005). A escolha da abordagem preditiva é normalmente feita conforme o nível de detalhamento desejado e disponibilidade de dados dendrométricos.

Os modelos em nível de povoamento, ou do tipo povoamento total, exprimem explicitamente a produção em unidade de área (Dias et al., 2005; Cruz et al., 2008). Tal abordagem preditiva não explica diretamente a variação do tamanho das árvores dentro das unidades de manejo, fato que não limita seu uso quando o destino da madeira é a produção de celulose ou energia (Campos \& Leite, 2017; Castro et al., 2013; Mendonça et al. 2014). Os melhores modelos são aqueles de densidade variável, considerada parte dinâmica de um sistema de equações (Salles et al., 2012). O modelo mais difundido no Brasil é o de Clutter, ajustado orginalmente para parcelas estabelecidas em povoamentos de Pinus taeda L. (Clutter, 1963). Este modelo tem as características de consistência para área basal (a estimativa projetada para a mesma idade resulta em valores idênticos aos observados) e de compatibilidade entre o crescimento e a produção (Campos \& Leite, 2017).

Os sistemas de predição implícita são aqueles que requerem informações básicas da produção e da estrutura do povoamento, como os modelos de distribuição de diâmetros (Dias et al., 2005; Scolforo et al., 2016). Embora sejam mais laboriosos no ajustamento e projeção que aqueles de povoamento total, são frequentemente usados para a obtenção de multiprodutos da madeira (Mendonça et al. 2014).

Um processo comum aos modelos de distribuição de diâmetros é o uso de funções de densidade de probabilidade (f.d.p) estatística para distribuir os diâmetros em classes de tamanho (Diamantopoulou et al., 2015). A função mais utilizada no setor florestal é a de Weibull (Campos \& Leite, 2017). Descrita por Weibull (1951), a função é capaz de descrever diversas distribuições uni-modais e seus parâmetros podem ser estimados sem integração numérica.

Os modelos de distribuição de diâmetros fornecem estimativas da quantidade de fustes, área basal e volume por hectare em cada classe de diâmetro nas idades presente e futura (Araújo Júnior, et al., 2010; Araújo Júnior, et al., 2012; Retslaff et al., 2012). A partir da aplicação de equações de volume, de taper ou de razão volumétrica permite a estimação da produção por hectare em cada classe (Campos \& Leite, 2017). A prognose é aplicada para cada intervalo por sistemas de equações que estimam os parâmetros da f.d.p. para idades futuras (Castro et al., 2013). A produção total é calculada pelo somatório da variável de interesse em todas essas classes.

Os modelos de árvores individuais fornecem informações mais detalhadas e tratam cada árvore como unidade básica para a predição, em vez de parcela (Dias et al., 2005; Soares et al., 2017). Demanda total controle nas medições das parcelas permanentes, inclusive da idade exata em que uma árvore morre (Campos \& Leite, 2017). Trata-se de uma abordagem preditiva amplamente empregada em florestas inequiâneas para investigar padrões de crescimento entre espécies e fitofisionomias (Soares et al., 2017). 
Embora a aplicação dos modelos de crescimento e produção em nível de povoamento seja uma prática comum na silvicultura brasileira, o uso daqueles de distribuição de diâmetros e, sobretudo, de árvores individuais, ainda, é pouco explorado em florestas equiâneas e monoespecíficas (Campos \& Leite, 2017; Castro et al., 2013). A carência de dados apropriados para o ajuste, maior quantidade de funções que compõem o modelo e falta de pesquisas que demonstrem a precisão são prováveis empecilhos para a adoção de abordagens preditivas mais detalhadas.

O objetivo do presente estudo foi avaliar três categorias de modelos de crescimento e produção para a estimativa volumétrica em povoamentos comerciais de eucalipto.

\section{MATERIAL E MÉTODOS}

\section{Caracterização da área de estudo}

O estudo foi realizado com dados de plantios clonais de Eucalyptus urophylla S. T. Blake, situados na porção central do estado de Minas Gerais, sendo a área de estudo dividida em quatro unidades de manejo florestal (UMF) que apresentam clima do tipo Cwa (Clima subtropical de inverno seco), conforme classificação de Köppen \& Geiger (1928). A UMF 1 está localizada sob as coordenadas planas (datum SIRGAS 2000, UTM, zona 23 S) 459437 de longitude e 7903580 de latitude. Possui média de temperatura e precipitação de $22,7^{\circ} \mathrm{C}$ e $1259 \mathrm{~mm}$, respectivamente. O solo da região é do tipo Latossolo Vermelho, caracterizados pela presença de teores elevados de óxidos de ferro. A UMF 2 está localizada sob as coordenadas planas (datum SIRGAS 2000, UTM, zona 23 S) 554991 de longitude e 7900318 de latitude. Possui média de temperatura e precipitação de $23,4^{\circ} \mathrm{C}$ e $1043 \mathrm{~mm}$, respectivamente. O solo da região é do tipo Latossolo Vermelho-Amarelo com presença do horizonte $B$ textural. A UMF 3 está localizada sob as coordenadas planas (datum SIRGAS 2000, UTM, zona 23 S) 567041 de longitude e 7957427 de latitude. Possui média de temperatura e precipitação de $23,4^{\circ} \mathrm{C}$ e $1043 \mathrm{~mm}$, respectivamente. O solo da região é do tipo Latossolo Vermelho-Amarelo com presença do horizonte B textural. A UMF 4 está localizada sob as coordenadas planas (datum SIRGAS 2000, UTM, zona 23 S) 486435de longitude e 7921081 de latitude. Possui média de temperatura e precipitação de $23,0^{\circ} \mathrm{C}$ e $1189 \mathrm{~mm}$, respectivamente. O solo da região é do tipo Latossolo Vermelho distrófico-típico.

\section{Descrição dos dados}

Os dados foram provenientes de 34 parcelas permanentes distribuídas em povoamentos estabelecidos com um clone de eucalipto, inseridos na região central de Minas Gerais. Foram definidas quatro unidades de manejo florestal (UMF), sendo lançadas: 11 parcelas na UMF1 ( $87,91 \mathrm{ha}) ; 6$ parcelas na UMF2 (42,74 ha); 10 parcelas na UMF3 ( $77,21 \mathrm{ha})$ e 7 parcelas na UMF4 (85,57 ha). As parcelas possuíam uma área de $400 \mathrm{~m}^{2}(20 \times 20 \mathrm{~m})$ e o espaçamento de plantio foi de $3,0 \times 2,5 \mathrm{~m}$.

O inventário florestal contínuo foi realizado nas idades de 24, 36, 48, 60 e 72 meses. Mensurou-se o diâmetro com casca de todos os fustes, tomado à altura de 1,30 $\mathrm{m}$ do solo $(D A P, \mathrm{~cm})$. Em cada parcela, foram tomadas a altura total $(H, \mathrm{~m})$ de 15 árvores e de 4 árvores dominantes (Hdom, m), de acordo conceito de Assmann (1970), que concerne à altura média das 100 árvores de maior diâmetro em um hectare.

As estimativas de volume com casca foram obtidas pelo ajuste do modelo de Schumacher e Hall (1933) linearizado. Foi utilizada uma equação volumétrica específica para cada UMF, fornecida pela empresa florestal (Tabela 1).

O índice do sítio (S) das UMF's foi estimado usando as medidas de altura dominante, a partir do estabelecimento de uma relação funcional de Hdom com a idade. A equação obtida foi rearranjada conforme o método da curva-guia (Clutter et al., 1983) para estimação do índice de sítio (idade-índice foi igual a 60 meses). 
Tabela 1. Equações de volume com casca $\left(\mathrm{V}, \mathrm{m}^{3}\right.$.árvore $\left.{ }^{-1}\right)$ de cada unidade de manejo florestal (UMF)

\begin{tabular}{cc}
\hline UMF & Equações \\
\hline 1 & $\operatorname{Ln}(V)=-9,8529+1,9212 \cdot \operatorname{Ln}(D A P)+0,9395 \cdot \operatorname{Ln}(H t)$ \\
2 & $\operatorname{Ln}(V)=-9,6953+1,8465 \cdot \operatorname{Ln}(D A P)+0,9754 \cdot \operatorname{Ln}(H t)$ \\
3 & $\operatorname{Ln}(V)=-9,6356+1,9982 \cdot \operatorname{Ln}(D A P)+0,8251 \cdot \operatorname{Ln}(H t)$ \\
4 & $\operatorname{Ln}(V)=-9,8927+1,7132 \cdot \operatorname{Ln}(D A P)+1,1522 \cdot \operatorname{Ln}(H t)$ \\
\hline
\end{tabular}

Em que: $L n=$ logaritmo neperiano.

$\operatorname{Ln}(\operatorname{Hdom})=\alpha_{0}+\alpha_{1}\left(\frac{1}{I}\right)+\operatorname{Ln}(\varepsilon)$

$\operatorname{Ln}(S)=\operatorname{Ln}(H d o m)-\alpha_{1}\left(\frac{1}{I}-\frac{1}{60}\right)+\operatorname{Ln}(\varepsilon)$

em que:

$\alpha_{0}$ e $\alpha_{1}=$ parâmetros do modelo; e

$\varepsilon=$ erro aleatório, $\varepsilon \sim \operatorname{NID}\left(0, \sigma^{2}\right)$.

As categorias de modelo de crescimento e produção avaliadas foram em nível de povoamento; de distribuição diamétrica e de árvores individuais. Os ajustes foram realizados empregando os dados de todas as UMF's.

\section{Modelo em nível de povoamento (MP)}

As estimativas dos parâmetros do modelo de Clutter (1963) foram obtidas pelo método dos mínimos quadrados, em dois estágios, com auxílio do programa Eviews 5.0 (IHS Global, 2004). O sistema equações simultâneas foi:

$$
\left\{\begin{array}{c}
\operatorname{Ln}\left(B_{2}\right)=\operatorname{Ln}\left(B_{1}\right)\left(\frac{I_{1}}{I_{2}}\right)+\varnothing_{0}\left(1-\frac{I_{1}}{I_{2}}\right)+\varnothing_{1}\left(1-\frac{I_{1}}{I_{2}}\right) S+\operatorname{Ln}(\varepsilon) \\
\operatorname{Ln}\left(V_{2}\right)=\varnothing_{2}+\varnothing_{3}\left(\frac{1}{I_{2}}\right)+\varnothing_{4} S+\varnothing_{5} \operatorname{Ln}\left(B_{2}\right)+\operatorname{Ln}(\varepsilon)
\end{array}\right.
$$

Em que:

$B_{1}$ e $B_{2}=$ áreas basais presente e futura $\left(\mathrm{m}^{2} . \mathrm{ha}^{-1}\right)$, respectivamente;

$I_{1}$ e $I_{2}=$ idades presente e futura (meses), respectivamente;

$V_{2}=$ volume com casca futuro $\left(\mathrm{m}^{3} \cdot \mathrm{ha}^{-1}\right)$;

$\varnothing_{0}$ e $\varnothing_{1}=$ parâmetros do modelo de área basal; e

$\varnothing_{2} \ldots \varnothing_{5}=$ parâmetros do modelo volumétrico.

\section{Modelo de distribuição diamétrica (MDD)}

Os dados foram agrupados em classes com intervalos regulares de 2,0 cm de diâmetro. Para modelar a frequência diamétrica por parcela foi adotada a função Weibull de dois parâmetros, com parâmetro de locação (a) fixado a zero. Esta decisão pautou-se na premissa de que o parâmetro locação possui baixa correlação com características do povoamento e sua exclusão facilita a projeção dos parâmetros de forma e escala (Rustagi, 1977; Nogueira, 2003; Binoti et al., 2010).

$$
f(x)=\left(\frac{\gamma}{\beta}\right)\left(\frac{x}{\beta}\right)^{\gamma-1} e^{-\left(\frac{x}{\beta}\right)^{\gamma}}
$$


Em que:

$\beta$ e $\gamma=$ parâmetro de escala e forma, respectivamente;

$x=$ centro de classe de diâmetro $(\mathrm{cm}), x \geq 0$.

O método de estimação dos parâmetros foi o da máxima verossimilhança, devido sua característica assintótica e por proporcionar estimativas mais confiáveis e com menor tendenciosidade (Nogueira et al., 2005; Leite et al., 2010; Diamantopoulou et al., 2015). Os parâmetros escala e forma foram obtidos para cada uma das parcelas disponíveis no conjunto de dados. Os ajustes foram realizados através de planilhas eletrônicas do software da Microsoft Office Excel®.

A redistribuição teórica dos diâmetros por classe, ou recuperação da distribuição diamétrica, foi concebida relacionando os parâmetros obtidos da função Weibull com características do povoamento, como a idade. Essas relações foram estabelecidas através do ajuste de um conjunto de modelos aplicando o método dos mínimos quadrados e o procedimento iterativo Quasi-Newton. Os ajustes foram efetuados no software Statistica 8.0 (StatSoft Inc., 2009).

$$
\begin{aligned}
& \operatorname{Ln}\left(y_{2}\right)=\operatorname{Ln}\left(y_{1}\right) e^{-\theta_{l}\left(I_{2}^{\theta_{2}}-I_{I}^{\theta_{2}}\right)}+\varepsilon \\
& \operatorname{dmax}_{2}=\operatorname{dmax} e_{1} e^{-\theta_{3}\left(I_{2}^{\theta_{4}}-I_{I}^{\theta_{4}}\right)}+\varepsilon \\
& \operatorname{Ln}\left(\beta_{2}\right)=\operatorname{Ln}\left(\beta_{1}\right) e^{-\theta_{5}\left(I_{2}^{\theta_{6}}-I_{I}^{\theta_{\sigma}}\right)}+\varepsilon \\
& N_{2}=N_{I} e^{-\theta_{7}\left(I_{2}^{\theta_{8}}-I_{I}^{\theta_{s}}\right)}+\varepsilon
\end{aligned}
$$

Em que:

$\gamma_{1}$ e $\gamma_{2}=$ parâmetros de forma da função Weibull em $I_{1} \mathrm{e} I_{2}$, respectivamente;

$I_{1}$ e $I_{2}=$ idades presente e futura (meses), respectivamente;

$\operatorname{dmax}_{1}$ e $\operatorname{dmax}_{2}=$ diâmetro máximo em $I_{1} \mathrm{e} I_{2}$, respectivamente;

$\beta_{1}$ e $\beta_{2}=$ parâmetros de escala da função Weibull em $I_{1}$ e $I_{2}$, respectivamente;

$N_{1}$ e $N_{2}=$ número de árvores.ha ${ }^{-1} \mathrm{em} I_{1}$ e $I_{2}$, respectivamente; e

$\theta_{1} \ldots \theta_{8}=$ parâmetros do conjunto de relações funcionais para redistribuição dos diâmetros.

A estrutura diamétrica futura foi projetada a partir da distribuição observada na idade presente. A altura total referente a cada centro de classe de diâmetro foi estimada após a seleção de uma equação hipsométrica, sendo testados quatro modelos citados por Nogueira (2003). Os modelos testados relacionaram a altura total com variáveis individuais da árvore e do povoamento.

$$
\begin{aligned}
& \operatorname{Ln}(H t)=\theta_{8}+\theta_{9} S+\theta_{10}\left(\frac{1}{D A P}\right)+\theta_{11} \operatorname{Ln}(I)+\varepsilon \\
& H t=\theta_{8}+\theta_{9} I+\theta_{10} D A P+\theta_{11} S+\varepsilon \\
& H t=\theta_{8}+\theta_{9} I+\theta_{10} S+\theta_{11} \operatorname{Ln}(I . D A P)+\varepsilon \\
& H t=\theta_{8}+\theta_{9} D A P+\theta_{10} S \cdot \operatorname{Ln}(I)+\varepsilon
\end{aligned}
$$

A produção total em volume com casca foi totalizada por unidade de área (hectare).

\section{Modelo de árvores individuais (MAI)}

A construção do modelo de árvores individuais envolveu a projeção das dimensões de cada árvore e a estimação de sobrevivência no decorrer dos anos. O modelo apresentado por 
Pienaar \& Shiver (1981), originalmente desenvolvido para descrever a sobrevivência vegetal, foi ajustado para a obtenção das estimavas futuras de $D A P, H$ e densidade do povoamento $(N)$. Ajustou-se esse modelo através do método dos mínimos quadrados e o procedimento iterativo Quasi-Newton.

$Y_{2}=Y_{1} e^{-\delta_{0}\left(I_{2}^{\delta_{l}}-I_{1}^{\delta_{l}}\right)}+\varepsilon$

Em que:

$Y_{1}$ e $Y_{2}=D A P, H$ ou Nem $I_{1}$ e $I_{2}$, respectivamente;

$I_{1}$ e $I_{2}=$ idades presente e futura (meses), respectivamente; e

$\delta_{0}$ e $\delta_{l}=$ parâmetros do modelo de Pienaar \& Shiver (1981).

\section{Avaliação dos modelos}

A precisão dos ajustes e projeções das parcelas foi avaliada através da raiz quadrada do erro médio (RQEM, \%), média das diferenças percentuais (MDP, \%), coeficiente de correlação de Pearson ( $\left.r_{Y \hat{Y}}\right)$ e análise gráfica dos resíduos percentuais, conforme Campos \& Leite (2017).

$$
\begin{aligned}
& \operatorname{RQEM}(\%)=\frac{100}{\bar{V}} \sqrt{\frac{\sum_{i=l}^{n}\left(V_{i}-\hat{V}_{i}\right)^{2}}{n}} \\
& \operatorname{MDP}(\%)=\frac{1}{n_{i=l}} \sum_{i=l}^{n}\left(\frac{\hat{V}_{i}-V_{i}}{V_{i}}\right) 100 \\
& r_{Y \hat{Y}}=\frac{\operatorname{cov}(V, \hat{V})}{\sqrt{S^{2}(V) S^{2}(\hat{V})}}
\end{aligned}
$$

Em que:

$V_{i}, \hat{V}_{i}$ e $\bar{V}=$ volumes com casca observado, estimado e médio $\left(\mathrm{m}^{3} \cdot \mathrm{ha}^{-1}\right)$;

$n=$ número de casos;

$S^{2}=$ variância;

cov = covariância.

Para avaliar a capacidade preditiva entre os modelos além dos períodos mensurados, o volume com casca das UMF's na idade de 24 meses foi projetado até 120 meses. O teste de $\mathrm{F}\left(H_{0}\right)$ Graybill (1976) foi aplicado para avaliar a aderência dos dados estimados aos observados. Inicialmente, foi realizada a análise do modelo linear $Y_{j}=\beta_{0}+\beta_{I} Y_{I}+\varepsilon$, sendo $Y_{j}=$ alturas estimadas e $Y_{I}=$ alturas observadas. A similaridade entre $Y_{I}$ e $Y_{j}$ foi verificada a partir do teste de hipótese, sendo $H_{0}=\beta^{\prime}=\left[\begin{array}{l}0 \\ 1\end{array}\right]$ "versus" $H_{a}=n \tilde{a} o H_{0}$. Para testar a hipótese, aplicou a fórmula, sob hipótese de normalidade:

$$
F\left(H_{0}\right)=\frac{(\beta-\theta)^{\prime}\left(Y_{l}^{\prime} Y_{l}\right)(\beta-\theta)}{2 Q M \operatorname{Res}} \sim F_{\alpha}(2, n-2 g l .)
$$

Em que:

$$
\begin{aligned}
& \beta=\left[\begin{array}{l}
\hat{\beta}_{0} \\
\hat{\beta}_{I}
\end{array}\right] ; \\
& \theta=\left[\begin{array}{l}
0 \\
1
\end{array}\right] ; \\
& Y_{I}^{\prime} Y_{I}=\left[\begin{array}{cc}
n & \sum Y_{I} \\
\sum Y_{I} & \sum Y_{I}^{2}
\end{array}\right] ; \mathrm{e}
\end{aligned}
$$


QMRes = quadrado médio do resíduo.

Assim, se $F\left(H_{0}\right) \geq F a(2, n-2 g /$.$) , a hipótese H_{0}$ é rejeitada. Caso $F\left(H_{0}\right)<F a(2, n-2 g /$.$) , a$ hipótese $H_{0}$ não é rejeitada, admitindo-se a identidade entre dois métodos avaliados, ou seja, $Y_{j}=Y_{l}$, considerando-se o nível a de significância.

Para diagnóstico do efeito significativo, adotou-se o nível de confiança de 95,0\% de probabilidade em todas as análises estatísticas.

\section{RESULTADOS E DISCUSSÃO}

As estatísticas de precisão apresentadas abaixo evidenciaram um bom desempenho da equação de altura dominante. A partir da premissa de que o índice de sítio é a altura dominante em uma idade índice, foi obtida uma equação de sítio para todas as UMF's. As estimativas de $\mathrm{S}$ foram utilizadas no ajuste do modelo de Clutter (1963).

$$
\begin{aligned}
& \operatorname{Ln}(H d)=3,6948-24,8863\left(\frac{1}{I}\right) ; \operatorname{RQEM}=11,83 \% ; M D P=0,33 \% \text { e } r_{Y \hat{Y}}=0,876^{*} ; \\
& \operatorname{Ln}(S)=\operatorname{Ln}(H d)-(-24,8863)\left(\frac{1}{I}-\frac{1}{60}\right)
\end{aligned}
$$

As equações obtidas para cada categoria de modelo e suas respectivas medidas de precisão estão apresentadas na Tabela 2. A qualidade dos ajustes foi satisfatória, a maioria com baixos valores de RQEM $(<10 \%)$, MDP próximos a zero e coeficientes de correlação relativamente altos $(>0,94)$ (Figura 1). Estes resultados são indícios de que as variáveis independentes utilizadas nas modelagens têm influência naquelas dependentes (Campos \& Leite, 2017).

\begin{tabular}{|c|c|c|c|c|}
\hline Modelos & Equações & $\begin{array}{l}\text { RQEM } \\
(\%)\end{array}$ & $\begin{array}{l}\text { MDP } \\
(\%)\end{array}$ & $r_{Y \hat{Y}}$ \\
\hline \multirow{2}{*}{ MP } & $\operatorname{Ln}\left(B_{2}\right)=\operatorname{Ln}\left(B_{1}\right)\left(\frac{I_{1}}{I_{2}}\right)+3,6360\left(1-\frac{I_{1}}{I_{2}}\right)$ & 5,76 & 1,31 & 0,971 * \\
\hline & $\operatorname{Ln}\left(V_{2}\right)=1,8849-13,6104\left(\frac{1}{I_{2}}\right)+0,0144 S+1,1379 \operatorname{Ln}\left(B_{2}\right)$ & 8,50 & 1,72 & 0,969 * \\
\hline \multirow{5}{*}{ MDD } & $\operatorname{Ln}\left(y_{2}\right)=\operatorname{Ln}\left(y_{1}\right) e^{-0,0038\left(I_{2}^{0,7339}-I_{1}^{0,7339}\right)}$ & 24,56 & 0,64 & 0,650 * \\
\hline & $\operatorname{dmax}_{2}=\operatorname{dmax}_{1} e^{-97,2887\left(I_{2}^{-1,5948}-I_{I}^{-1,5948}\right)}$ & 3,43 & $-0,15$ & $0,969 *$ \\
\hline & $\operatorname{Ln}\left(\beta_{2}\right)=\operatorname{Ln}\left(\beta_{1}\right) e^{-77,975 I\left(I_{2}^{-1,8457}-I_{I}^{-1,8457}\right)}$ & 3,78 & 0,01 & $0,962^{*}$ \\
\hline & $N_{2}=N_{l} e^{-269,9613\left(I_{2}^{0,000029}-I_{l}^{0,000029}\right)}$ & 0,68 & $-0,01$ & $0,998 *$ \\
\hline & $\operatorname{Ln}(H t)=1,7395+0,0159 \cdot S-6,4763\left(\frac{1}{D A P}\right)+0,3637 \cdot \operatorname{Ln}(I)$ & 6,84 & 0,29 & 0,964 * \\
\hline \multirow{3}{*}{ MAI } & $D A P_{2}=D A P_{1} e^{-121,7257\left(I_{2}^{-1,6956}-I_{1}^{-1,6996}\right)}$ & 4,13 & $-0,20$ & $0,974 *$ \\
\hline & $H t_{2}=H t_{l} e^{-l 35,3378\left(I_{2}^{-I, 6246}-I_{I}^{-I, 6246}\right)}$; & 5,73 & $-0,15$ & $0,948 *$ \\
\hline & $N_{2}=N_{I} e^{-269,9613\left(I_{2}^{0,000029}-I_{I}^{0,000029}\right)}$ & 0,68 & $-0,01$ & $0,998 *$ \\
\hline
\end{tabular}

Tabela 2. Equações obtidas para as três categorias de modelo avaliadas

$\mathrm{MP}=$ modelo em nível de povoamento (Clutter, 1963); $\mathrm{MDD}=$ modelo de distribuição diamétrica (Weibull-2P); MAI = modelo de árvores individuais (Pienaar \& Shiver, 1981); RQEM = raiz quadrada do erro médio; MDP = média das diferenças percentuais e $r_{Y \hat{Y}}=$ coeficiente de correlação de Pearson. *correlação de Pearson significativa $(p<0,05)$. 
$\left(\operatorname{RQEM}=8,50 \% ; \mathrm{MDP}=1,72 \%\right.$ e $\left.r_{Y \hat{Y}}=0,969^{*}\right)$

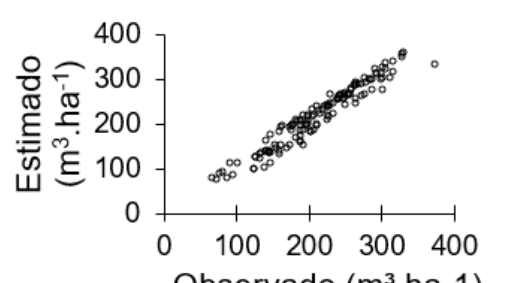

Observado $\left(\mathrm{m}^{3}\right.$.ha- 1$)$

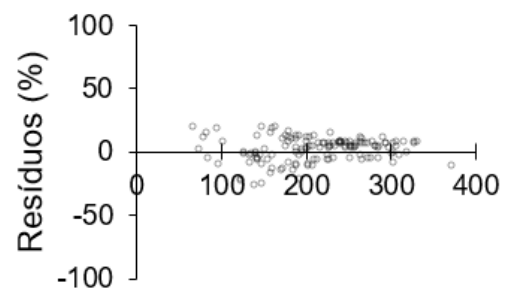

Modelo de distribuição diamétrica - MDD

$\left(\mathrm{RQEM}=7,50 \% ; \mathrm{MDP}=-1,01 \%\right.$ e $\left.r_{Y \hat{Y}}=0,973^{*}\right)$
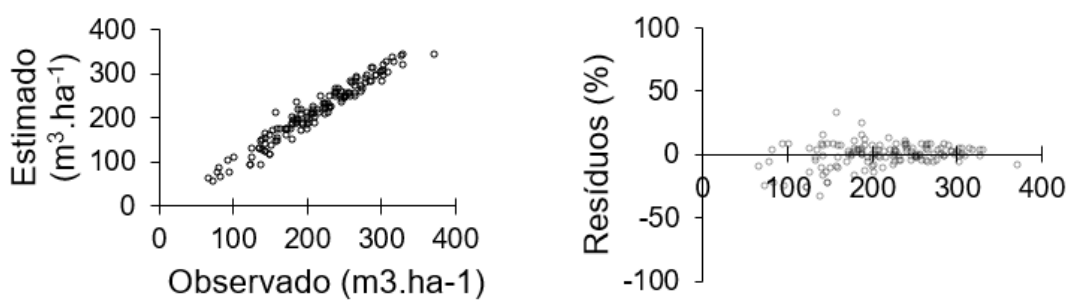

Modelo de árvores individuais - MAI

$\left(\operatorname{RQEM}=8,23 \% ;\right.$ MDP $=-0,74 \%$ e $\left.r_{Y \hat{Y}}=0,968^{*}\right)$
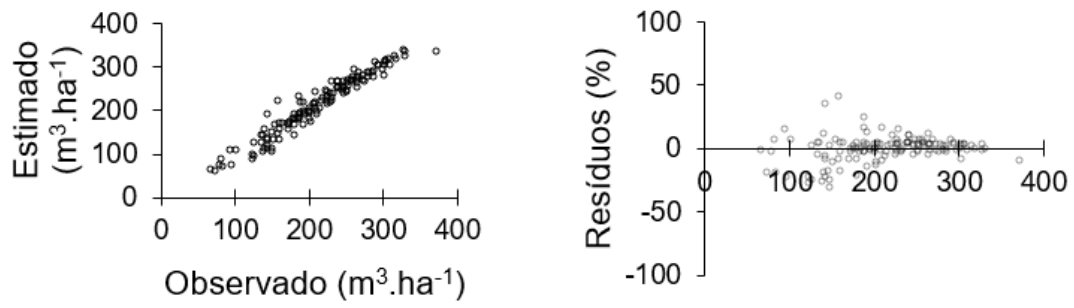

Figura 1. Distribuição dos resíduos percentuais e da relação entre volumes com casca estimados e observados para três categorias de modelo.

Os sinais associados aos parâmetros do sistema de equações simultâneas de Clutter (1963) devem ser analisados com atenção. $O$ sinal de $\varnothing_{3}$, associado ao inverso da idade, foi negativo e está coerente com a literatura (Campos \& Leite, 2017). Por outro lado, verificou-se a ocorrência de valor negativo no parâmetro relacionado ao índice de sítio na equação de área basal. $O \varnothing_{1}$ foi negativo mesmo substituindo $S$ por $\operatorname{LnB_{1}},\left(\operatorname{Ln} B_{1}\right)^{2}$ ou $H d$, resultando em inconsistência biológica (Salles et al., 2012; Castro et al., 2013). Logo, optou-se por remover o termo $\left[\left(1-\frac{I_{1}}{I_{2}}\right) S\right]$ do respectivo modelo, conforme sugerido por Campos \& Leite (2017).

Todos os parâmetros do modelo em nível de povoamento foram estatisticamente significativos pelo teste $t(p<0,05)$. Os erros das estimativas variaram de $-18,48$ a $12,21 \%$ para área basal e de - 25,66 a 20,33\% para o volume com casca. Esses valores estão de acordo com o verificado para plantações de eucalipto não desbastadas por Castro et al. (2013); Castro et al. (2015) e Azevedo et al. (2016). Uma leve tendência à superestimação nos maiores volumes foi observada no MP (Figura 1). Fato que corrobora com os resultados obtidos com o ajuste de Clutter (1963) por Demolinari et al. (2007) e Gonçalves et al. (2016). Segundo Gonçalves et al. (2016), a superestimativa volumétrica em idades avançadas é uma característica estatística inerente ao próprio modelo. 
As equações empregadas durante a redistribuição teórica dos diâmetros por classe, que estimam os parâmetros da função Weibull-2P, não foram tendenciosos e tiveram comportamento homocedástico. O MDD foi a categoria com menor RQEM $(7,50 \%)$ e maior coeficiente de correlação $\left(r_{Y \hat{Y}}=0,973\right)$, com erros variando de $-33,23$ a 33,58\% para o volume com casca (Figura 1).

A equação menos precisa obtida para o MDD foi aquela que concerne ao parâmetro de forma $\left(\gamma_{2}\right)$ (Tabela 2). Essa equação apresentou os maiores desvios (RQEM =24,56\%) e menor correlação $\left(r_{\hat{Y Y Y}}=0,650\right)$, porém baixa média residual (MDP $\left.=0,64 \%\right)$. Pouca precisão no ajuste de modelos que estimam $\gamma_{2}$ já foram relatadas por Nogueira et al. (2005); Binoti et al. (2014) e Azevedo et al. (2016). A maioria dos MDD's têm apresentado resultados satisfatórios, apenas no que refere ao parâmetro de escala (Binoti et al., 2014). Além disso, a baixa correlação de $\gamma_{2}$ com características do povoamento não prejudicou a qualidade de ajustamento do MDD; a aderência das estimativas volumétricas aos dados observados foi constatada pelo teste $\mathrm{F}$ Graybill.

A equação da sobrevivência apresentou um bom ajuste (valores em módulo de RQEM e MDP foram inferiores a 1\% e o coeficiente de correlação próximo ao seu valor máximo, de 1). A mortalidade natural das árvores foi pequena, diminuindo ligeiramente o número de árvores ao longo dos anos. Um aspecto importante que deve ser salientado é o de que a estimativa da quantidade de árvores mortas é de difícil previsibilidade, aleatório e com causas não complementarmente identificadas em uma unidade de manejo ou povoamento (CrecenteCampo et al., 2009; Castro et al., 2013).

A partir dos valores observados e estimados de volume com casca, o MAl apresentou o menor MDP $(|-0,74 \%|)$ (Figura 1); os erros das estimativas variaram de $-30,63$ a $41,55 \%$ para o volume. A amplitude de variação do $D A P$ e $H$ foram de $12,4 \mathrm{~cm}$ e $17,6 \mathrm{~m}$ aos 24 meses, de $14,8 \mathrm{~cm}$ e $23,3 \mathrm{~m}$ aos 36 meses, de 17,9 cm e 25,7 m aos 48 meses, de $18,9 \mathrm{~cm}$ e $25,6 \mathrm{~m}$ aos 60 meses e de $19,1 \mathrm{~cm}$ e 25,9 m aos 72 meses de idade, respectivamente. Mesmo que a variabilidade no tamanho das árvores tenda a ser crescente com a idade em povoamentos equiâneos monoespecíficos (Binkley et al., 2002; Soares et al., 2016, 2017), essa categoria foi mais precisa nos maiores volumes (menor dispersão dos resíduos percentuais). Estimativas volumétricas precisas, sobretudo em idades avançadas, são desejáveis para o manejo florestal, em que se busca maior precisão das projeções futuras de volume e de árvores com maior valor comercial.

A precisão das estimativas volumétricas oscilou entre as categorias de modelo e entre as idades projetadas. Os erros tenderam a ser menores à medida que reduziu o intervalo de projeção (Tabela 3), corroborando com o observado por Nogueira et al. (2005) e Araújo Júnior et al. (2010). O maior valor de RQEM foi verificado na predição de 24 para 72 meses do MDD. Para essas idades, tanto o MDD quanto o MP tenderam a superestimar a média do volume (MDP de $7,71 \%$ e $5,06 \%$, respectivamente).

A aderência das estimativas de volume com casca projetado a partir de 24 meses foi verificada pelo teste $F$ Graybill. As três categorias estimaram com precisão os volumes futuros (Figura 2). Entretanto, uma pequena tendência à superestimação da média do volume foi observada aos 72 meses quando se projetou com MP e MDD. Essa superestimação, provavelmente, relaciona-se ao comportamento sigmoidal da curva de produção e aos pontos de inflexão e assíntota em idades superiores à observadas, sendo melhor representadas pelo MAI. Apesar dos elevados valores de RQEM (de 16,50 a 23,35), a projeção com MAl a partir de 24 meses forneceu estimativas de volume sem tendências.

O comportamento do volume além da última idade inventariada (72 meses) deve ser visto com cautela. Nas condições de estudo, a projeção volumétrica com o MP além de 72 meses foi próxima à obtida com MDD (Figura 2). A modelagem de árvores individuais por regressão mostrou ser uma eficiente ferramenta de prognose. O MP e MDD, embora proporcione informações menos detalhadas, suas estimativas foram confiáveis para a projeção futura do volume com casca por hectare. 
Tabela 3. Estatísticas utilizadas para avaliar as diferenças entre produções totais observadas e estimadas por três categorias de modelos

\begin{tabular}{|c|c|c|c|c|c|c|c|c|c|}
\hline \multirow{2}{*}{$\begin{array}{c}\text { Idade de } \\
\text { projeção (meses) }\end{array}$} & RQEM (\%) & $\begin{array}{c}\text { MDP } \\
(\%)\end{array}$ & $r_{Y \hat{Y}}$ & $\begin{array}{c}\text { RQE } \\
\text { M (\%) }\end{array}$ & $\begin{array}{c}\text { MDP } \\
(\%)\end{array}$ & $r_{Y \hat{Y}}$ & $\begin{array}{c}\text { RQEM } \\
\text { (\%) }\end{array}$ & $\begin{array}{c}\text { MDP } \\
(\%)\end{array}$ & $r_{Y \hat{Y}}$ \\
\hline & \multicolumn{3}{|c|}{----- MP ------ } & \multicolumn{3}{|c|}{----- MDD ------ } & \multicolumn{3}{|c|}{------ MAI ------ } \\
\hline 24 para 36 & 13,64 & $-5,20$ & $0,901 *$ & 14,72 & $-2,49$ & $0,914^{*}$ & 16,50 & $-1,22$ & $0,887 *$ \\
\hline 24 para 48 & 12,37 & $-5,77$ & $0,835^{\star}$ & 21,71 & $-3,83$ & $0,857 *$ & 21,67 & $-4,47$ & 0,831 * \\
\hline 24 para 60 & 10,61 & 1,06 & 0,82 & 23,40 & 0,23 & $0,848 *$ & 21,97 & $-3,41$ & $0,822 *$ \\
\hline 24 para 72 & 13,38 & 7,71 & $0,786^{*}$ & 27,13 & 5,06 & $0,805^{\star}$ & 23,35 & $-2,29$ & $0,778 *$ \\
\hline 36 para 48 & 7,21 & 0,35 & $0,947 *$ & 8,10 & $-5,09$ & $0,967 *$ & 10,72 & $-3,84$ & $0,964 *$ \\
\hline 36 para 60 & 9,12 & 6,54 & $0,938^{*}$ & 7,78 & $-1,08$ & $0,966^{*}$ & 11,21 & $-2,77$ & 0,950 * \\
\hline 36 para 72 & 15,03 & 12,90 & $0,879 *$ & 11,89 & 3,37 & $0,918 *$ & 13,56 & $-1,60$ & $0,894 *$ \\
\hline 48 para 60 & 6,96 & 5,76 & $0,968^{*}$ & 3,19 & $-0,35$ & $0,988^{*}$ & 3,27 & 1,15 & $0,986 *$ \\
\hline 48 para 72 & 13,11 & 12,25 & $0,932 *$ & 6,52 & 4,02 & $0,958 *$ & 5,92 & 2,12 & $0,956 *$ \\
\hline 60 para 72 & 7,90 & 5,98 & $0,952 *$ & 5,99 & 3,88 & $0,966 *$ & 4,44 & 0,95 & $0,973 *$ \\
\hline
\end{tabular}

MP = modelo em nível de povoamento (Clutter, 1963); MDD = modelo de distribuição diamétrica (Weibull-2P); MAI = modelo de árvores individuais (Pienaar \& Shiver, 1981); RQEM = raiz quadrada do erro médio; MDP = média das diferenças percentuais e $r_{Y \hat{Y}}=$ coeficiente de correlação de Pearson. * correlação de Pearson significativa $(p<0,05)$.

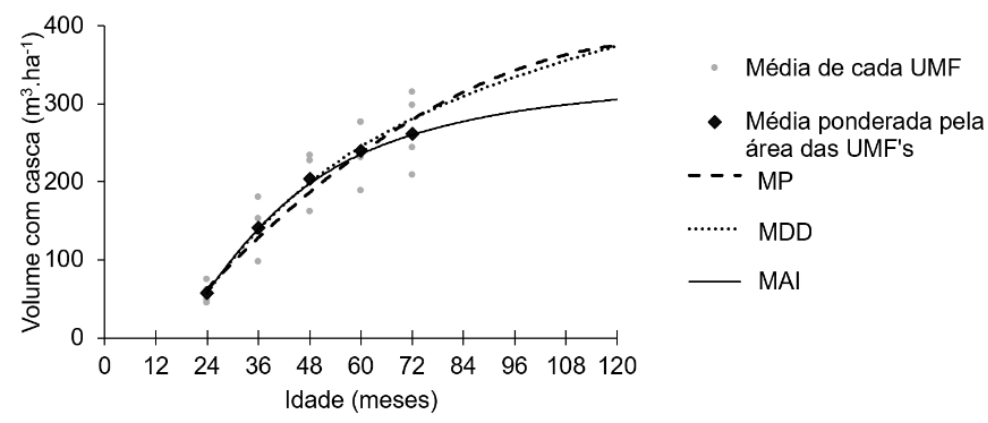

Figura 2. Estimativa e tendência de produção em volume com casca de povoamentos de Eucalyptus, obtidas por três categorias de modelo.

\section{CONCLUSÃO}

Os ajustes dos modelos em nível de povoamento, de distribuição de diâmetros e de árvores individuais proporcionam estimativas confiáveis da produção em volume com casca.

O modelo em nível de árvores individuais é mais preciso e consistente na estimativa do volume com casca por hectare.

O modelo em nível de árvores individuais fornece estimativas mais detalhadas em relação aquele de distribuição diamétrica e em nível de povoamento, consecutivamente.

\section{REFERÊNCIAS}

Araújo Júnior, C. A. A., Leite, H. G., Binoti, D. H. B., Castro, R. V. O., Binoti, M. L. M. S., \& Marcatti, G. E. (2012). Uso da função Weibull para descrever a área basal por classe de diâmetro de um povoamento de eucalipto. Scientia Forestalis, 40(95), 401-406.

Araújo Júnior, C. A. A., Nogueira, G. S., Oliveira, M. L. R., Miranda, R. O. V., Castro, R. V. O., \& Pelli, E. (2010). Projeção da distribuição diamétrica de povoamentos de eucalipto em diferentes amplitudes de classe. Pesquisa Agropecuária Brasileira, 45(11), 1275-1281. http://dx.doi.org/10.1590/S0100204X2010001100007.

Assmann, E. (1970). The principles of forest yield study. New York: Pergamon.

Azevedo, G. B., Oliveira, E. K. B., Azevedo, G. T. O., Buchmann, H. M., Miguel, E. P., \& Rezende, A. V. (2016). Modelagem da produção em nível de povoamento e por distribuição diamétrica em plantio de eucalipto. Scientia Forestalis, 44(110), 383-392. http://dx.doi.org/10.18671/scifor.v44n110.11. 
Binkley, D., Stape, J. L., Ryan, M. G., Barnard, H. R., \& Fownes, J. (2002). Age-related decline in forest ecosystem growth: an individual-tree, stand-structure hypothesis. Ecosystems, 5(1), 58-67. http://dx.doi.org/10.1007/s10021-001-0055-7.

Binoti, D. H. B., Leite, H. G., Nogueira, G. S., Silva, M. L. M., Garcia, S. L. R., \& Cruz, J. P. (2010). Uso da função Weibull de três parâmetros em um modelo de distribuição diamétrica para plantios de eucalipto submetidos a desbaste. Revista Árvore, 34(1), 147-156. http://dx.doi.org/10.1590/S010067622010000100016.

Binoti, M. L. M. S., Binoti, D. H. B., Leite, H. G., Silva, A. A. L., \& Pontes, C. (2014). Utilização de redes neurais artificiais para a projeção da distribuição diamétrica de povoamentos equiâneos. Revista Árvore, 38(4), 747-754. http://dx.doi.org/10.1590/S0100-67622014000400018.

Campos, J. C. C., \& Leite, H. G. (2017). Mensuração florestal: perguntas e respostas (5. ed.). Viçosa: UFV.

Castro, R. V. O., Cunha, A. B., Silva, L. V., Leite, H. G., \& Silva, A. A. L. (2015). Modelagem do crescimento e produção para um povoamento de Eucalyptus utilizando dois métodos para quantificação do índice de local. Scientia Forestalis, 43(105), 83-90.

Castro, R. V. O., Soares, C. P. B., Martins, F. B., \& Leite, H. G. (2013). Crescimento e produção de plantios comerciais de eucalipto estimados por duas categorias de modelos. Pesquisa Agropecuária Brasileira, 48(3), 287-295. http://dx.doi.org/10.1590/S0100-204X2013000300007.

Clutter, J. L. (1963). Compatible growth and yield models for Loblolly Pine. Forest Science, 9(3), 354-371.

Clutter, J. L., Forston, J. C., Pienaar, L. V., Brister, G. H., \& Bailey, R. L. (1983). Timber management: a quantitative approach. New York: Wiley.

Crecente-Campo, F., Marshall, P., \& Rodríguez-Soalleiro, R. (2009). Modelling non-catastrophic individual-tree mortality for Pinus radiata plantations in northwestern Spain. Forest Ecology and Management, 257(6), 1542-1550. http://dx.doi.org/10.1016/j.foreco.2009.01.007.

Cruz, J. P., Leite, H. G., Soares, C. P. B., Campos, J. C. C., Smit, L., Nogueira, G. S., \& Oliveira, M. L. R. (2008). Modelos de crescimento e produção para plantios comerciais jovens de Tectonagrandis em Tangará da Serra, Mato Grosso. Revista Árvore, 32(5), 821-828. http://dx.doi.org/10.1590/S010067622008000500006.

Davis, L. S., Johnson, K. N., Bettinger, P., \& Howard, T. E. (2005). Forest management: to sustain ecological, economic, and social values (4th ed.). Illinois: Waveland.

Demolinari, R. A., Soares, C. P. B., Leite, H. G., \& Souza, A. L. (2007). Crescimento de plantios clonais de eucalipto não desbastados na região de Monte Dourado (PA). Revista Árvore, 31(3), 503-512. http://dx.doi.org/10.1590/S0100-67622007000300016.

Diamantopoulou, M. J., Özçelik, R., Crecente-Campo, F., \& Eler, Ü. (2015). Estimation of Weibull function parameters for modelling tree diameter distribution using least squares and artificial neural networks methods. Biosystems Engineering, 133, 33-45. http://dx.doi.org/10.1016/j.biosystemseng.2015.02.013.

Dias, A. N., Leite, H. G., Campos, J. C. C., Couto, L., \& Carvalho, A. F. (2005). Emprego de um modelo de crescimento e produção em povoamentos desbastados de eucalipto. Revista Árvore, 29(5), 731-739. http://dx.doi.org/10.1590/S0100-67622005000500008.

Gonçalves, J. C., Carvalho, S. P. C., Oliveira, A. D., \& Gomide, L. R. (2016). Comparação dos modelos prognósticos de Clutter e da função logística. Pesquisa Florestal Brasileira, 36(87), 311-317. http://dx.doi.org/10.4336/2016.pfb.36.87.1230.

Graybill, F. A. (1976). Theory and application of linear model. Belmont: Duxbury.

IHS Global. (2004). Eviews 5: quantitative micro software. Version 5. Irvine: IHS Global.

Köppen, W., \& Geiger, R. (1928). Klimate der Erde (Wall-map $150 \mathrm{~cm} \times 200 \mathrm{~cm}$ ). Gotha: Verlag Justus Perthes.

Leite, H. G., Binoti, D. H. B., Guimarães, D. P., Silva, M. L. M., \& Garcia, S. L. R. (2010). Avaliação do ajuste das funções Weibull e hiperbólica a dados de povoamento de eucalipto submetidos a desbaste. Revista Árvore, 34(2), 305-311. http://dx.doi.org/10.1590/S0100-67622010000200013.

Mendonça, A. R., Calegario, N., Silva, G. F., Souza, A. L., Trugilho, P. F., Carvalho, S. P. C., \& Possato, E. L. (2014). Modelagem da produção de sortimentos em povoamentos de eucalipto. Cerne, 20(4), 587594. http://dx.doi.org/10.1590/01047760201420041366.

Nascimento, F. A. F., Dias, A. N., Figueiredo Filho, A., Miranda, G. M., \& Arce, J. E. (2015). Sistema de crescimento e produção para povoamentos de Pinus taeda na região norte de Santa Catarina. Cerne, 21(2), 235-242. http://dx.doi.org/10.1590/01047760201521021494. 
Nogueira, G. S. (2003). Modelagem do crescimento e da produção de povoamentos de Eucalyptus sp. e de Tectonagrandis submetidos a desbaste (Tese de doutorado). Universidade Federal de Viçosa, Viçosa.

Nogueira, G. S., Leite, H. G., Campos, J. C. C., Carvalho, A. F., \& Souza, A. L. (2005). Modelo de distribuição diamétrica para povoamentos de Eucalyptus sp. submetidos a desbaste. Revista Árvore, 29(4), 579589. http://dx.doi.org/10.1590/S0100-67622005000400010.

Pereira, J. C., Dias, P. A. S., Mergulhão, R. C., Thiersch, C. R., \& Faria, L. C. (2016). Modelo de crescimento e produção de Clutter adicionado de uma variável latente para predição do volume em um plantio de Eucalyptus urograndis com variáveis correlacionadas espacialmente. Scientia Forestalis, 44(110), 393-403. http://dx.doi.org/10.18671/scifor.v44n110.12.

Pienaar, L. V., \& Shiver, B. D. (1981). Survival functions for site prepared slash pine plantations in the flat woods of Georgia Northern Florida. Southern Journal Forestry, 5(2), 59-62. http://dx.doi.org/10.1093/sjaf/5.2.59.

Retslaff, F. A. S., Figueiredo Filho, A., Dias, A. N., Bernett, L. G., \& Figura, M. A. (2012). Prognose do crescimento e da produção em classes de diâmetro para povoamentos desbastados de Eucalyptus grandis no sul do Brasil. Revista Árvore, 36(4), 719-732. http://dx.doi.org/10.1590/S010067622012000400013

Rustagi, K. P. (1977). Predicting stand structure in even aged stands. In Proceedings of the Growth Models for Long Term Forecasting of Timber Yields (pp. 1-16). Vienna: IUFRO.

Salles, T. T., Leite, H. G., Oliveira Neto, S. N. O., Soares, C. P. B., Paiva, H. N., \& Santos, F. L. (2012). Modelo de Clutter na modelagem de crescimento e produção de eucalipto em sistemas de integração lavoura-pecuária-floresta. Pesquisa Agropecuária Brasileira, 47(2), 253-260. http://dx.doi.org/10.1590/S0100-204X2012000200014.

Schumacher, F. X., \& Hall, F. S. (1933). Logarithmicexpressionoftimber-tree volume. Journal of Agricultural Research, 47(9), 719-734.

Scolforo, H. F., Castro Neto, F., Scolforo, J. R. S., Burkhart, H., Mctague, J. P., Raimundo, M. R., Loos, R. A., Fonseca, S., \& Sartório, R. C. (2016). Modelling dominant height growth of eucalyptus plantations with parameters conditioned to climatic variations. Forest Ecology and Management, 380, 182-195. http://dx.doi.org/10.1016/j.foreco.2016.09.001.

Soares, A. A. V., Leite, H. G., Cruz, J. P., \& Forrester, D. I. (2017). Development of stand structural heterogeneity and growth dominance in thinned Eucalyptus stands in Brazil. Forest Ecology and Management, 384, 339-346. http://dx.doi.org/10.1016/j.foreco.2016.11.010.

Soares, A. A. V., Leite, H. G., Souza, A. L., Silva, S. R., Lourenço, H. M., \& Forrester, D. I. (2016). Increasing stand structural heterogeneity reduces productivity in Brazilian Eucalyptus monoclonal stands. Forest Ecology and Management, 373, 26-32. http://dx.doi.org/10.1016/j.foreco.2016.04.035.

StatSoft Inc. (2009). STATISTICA (data analysis software system), version 8. Tulsa, OK.

Trincado, G., Quezada P, R., \& von Gadow, K. (2003). A comparison of two stand table projection methods for young Eucalyptus nitens (Maiden) plantations in Chile. Forest Ecology and Management, 180(1-3), 443-451. http://dx.doi.org/10.1016/S0378-1127(02)00611-4.

Vanclay, J. K. (1994). Modelling forest growth and yield: applications to mixed tropical forests. Wallingford: School of Environmental Science and Management Papers.

Weibull, W. (1951). A statistical distribution function of wide applicability. Journal of Applied Mechanics, $18,293-297$.

Contribuição dos autores: TMAP: conceptualization, data curation, formal analysis, investigation, methodology, visualization, writing - original draft. BOL: investigation, data curation, formal analysis, methodology, writing review \& editing. GSN: conceptualization, supervision, investigation, writing - review \& editing. PHA: resources, writing - review \& editing. EBG: writing - review \& editing. MLRO: writing - review \& editing. 